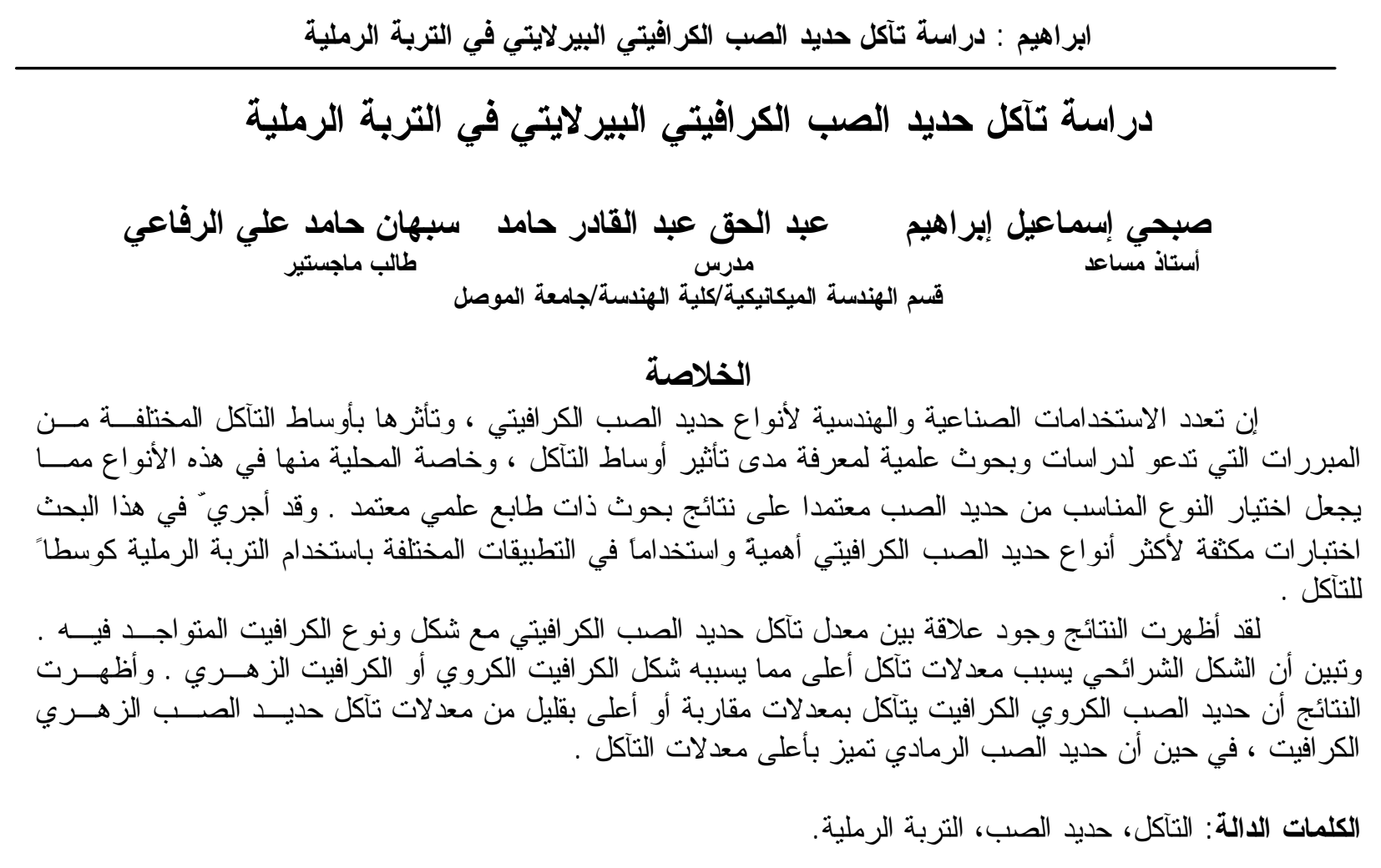

\title{
Studying the Corrosion of Graphite Containing pearlitic Cast Irons in Sandy Soil
}

\author{
Sobhi I. Ibrahim Abdulhaqq A. Hamid Sabhan H. AL-Rifay \\ Assistant Professor Lecturer M.Sc. Student \\ Mechanical Dept.-College of Engineering-Mosul University
}

\begin{abstract}
Wide range of industrial and engineering applications for graphite containing cast irons and the harmful effects of corrosion environments on these important engineering materials, make it necessary for continuous Scientific research in this field. In the current study, performed corrosion tests on Pearlitic cast irons using the sandy soil as the corrosion environment .

The results obtained show a clear relationship between corrosion rate of graphite containing cast iron with shape of graphite. The graphite flakes in cast iron appeared to cause high corrosion rates compared with spheroidal or rosette graphites. The results also show that corrosion rates of spheroidal cast iron are slightly higher than malleable cast iron. The corrosion rates of grey cast iron represent the highest when compared with those of spheroidal and malleable cast iron.
\end{abstract}

Keywords: Corrosion, Cast Iron, Sandy Soil 
\begin{tabular}{ccccc} 
Al-Rafidain Engineering & Vol.18 & No.2 & April 2010 \\
\hline المقدمة & & & &
\end{tabular}

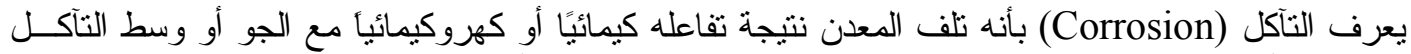

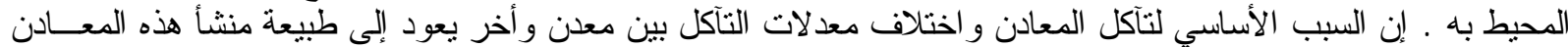

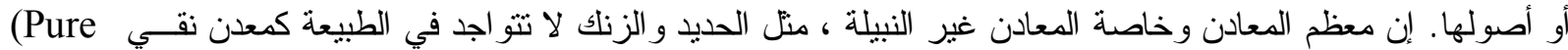

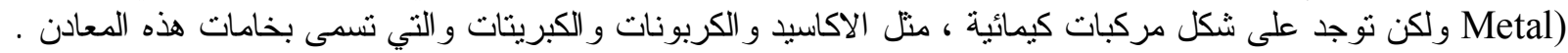

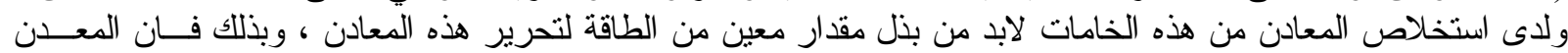

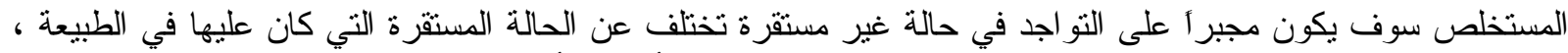

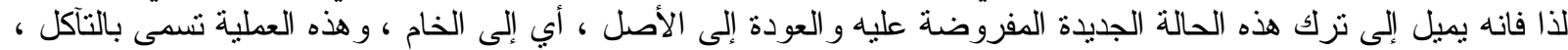

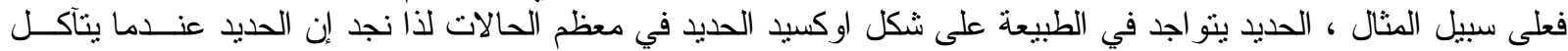

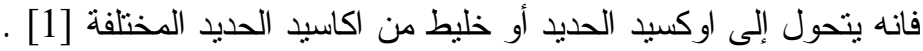

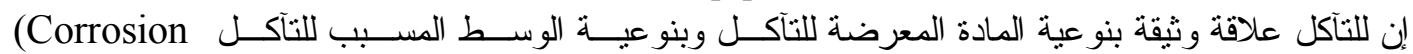

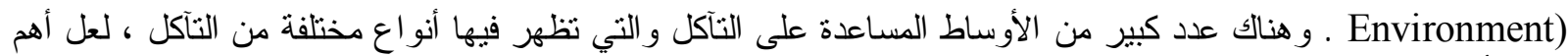

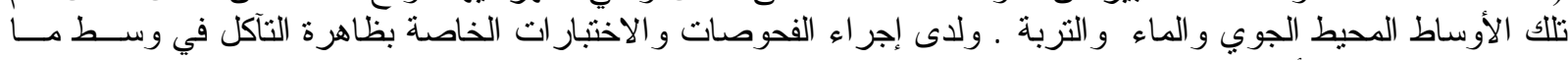

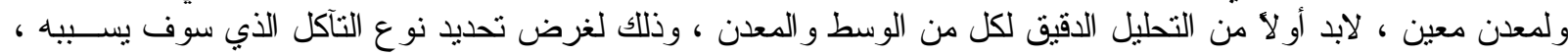

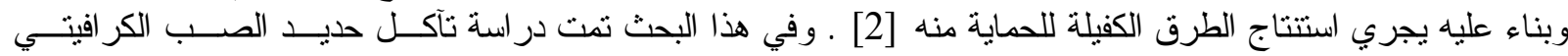

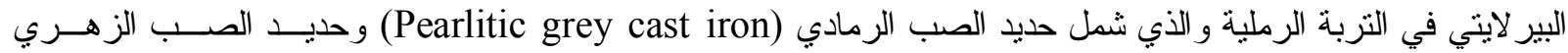

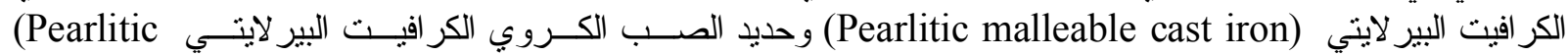

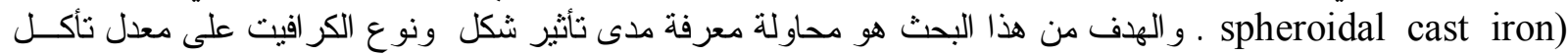

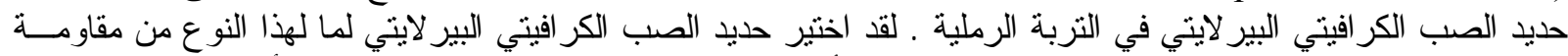

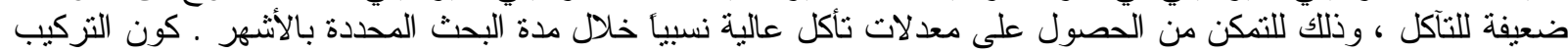

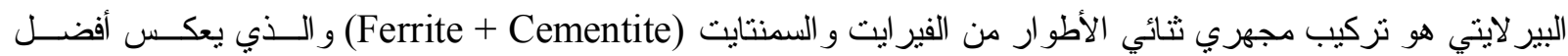

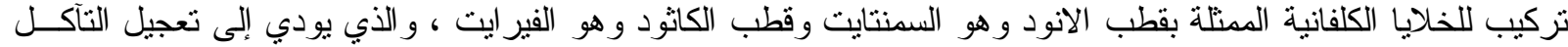

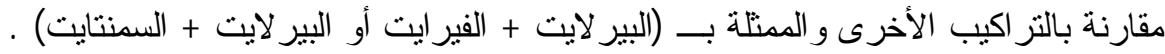

الار اسات السابقة

هنالك العديد من الدراسات و البحوث تتاولت در اسة تآكل حديد الصبّ الكر افيتي ومسبباته ، وكيفية الســبطرة

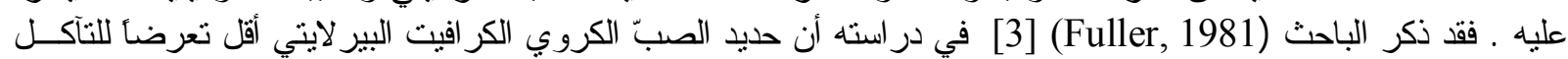

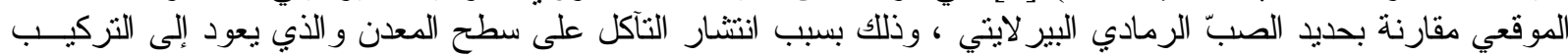

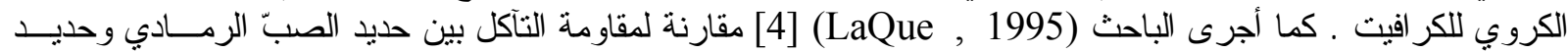

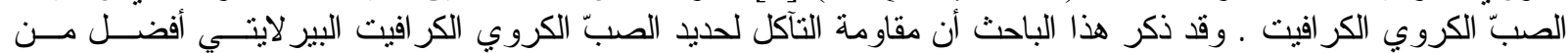

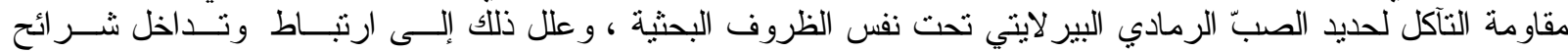
(Flakes)

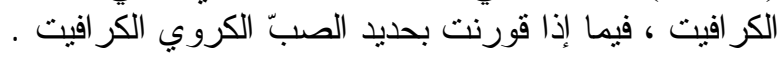

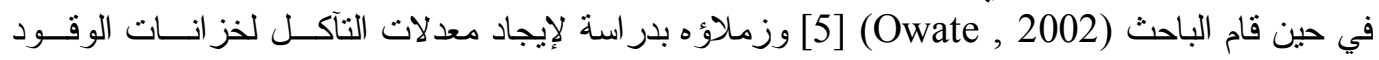

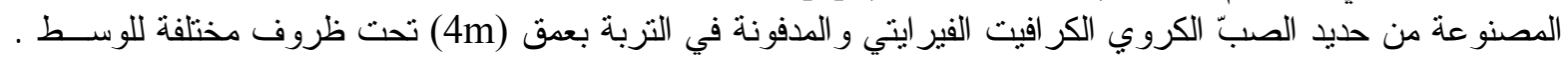

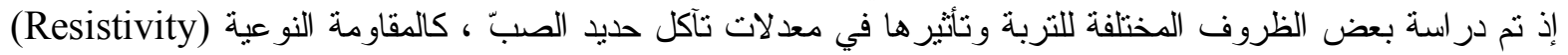

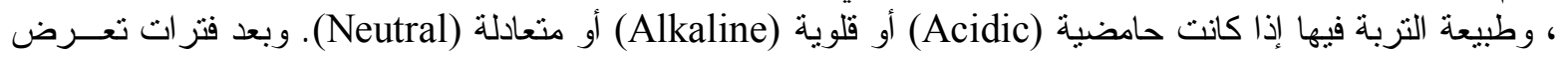

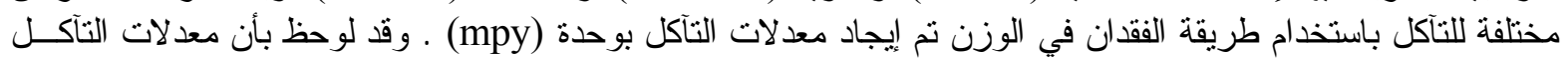

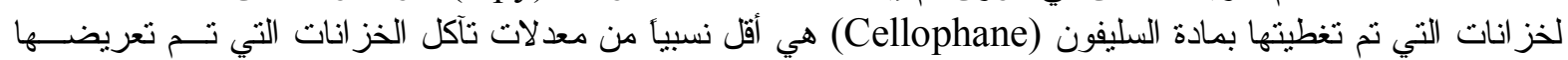

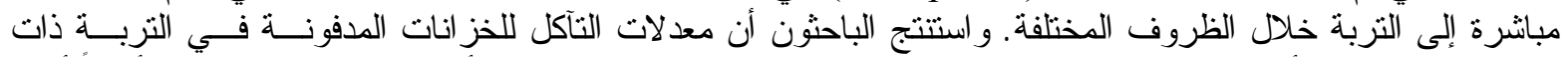

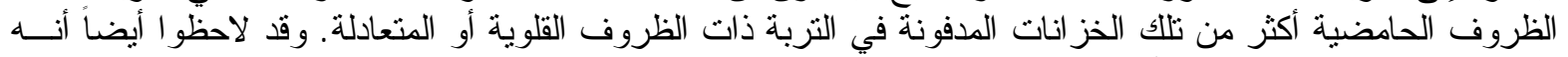

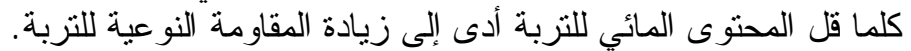

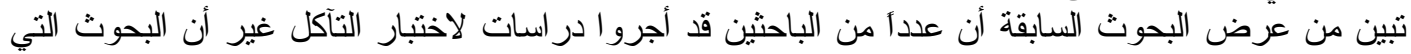

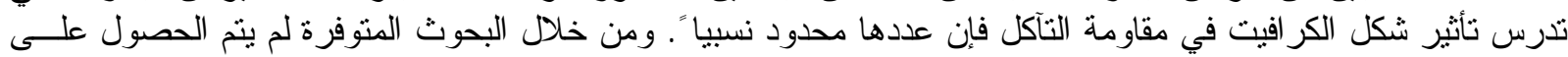




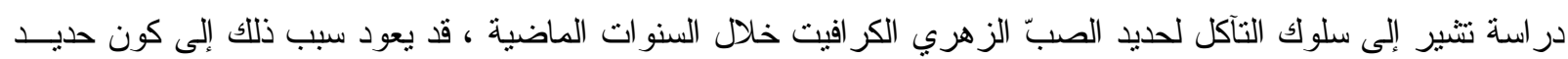

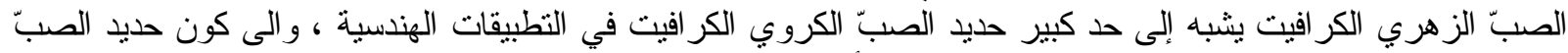

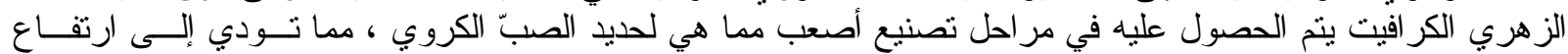

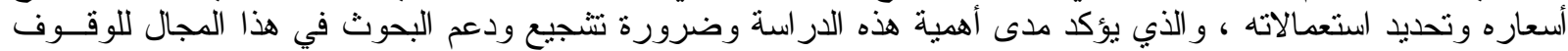

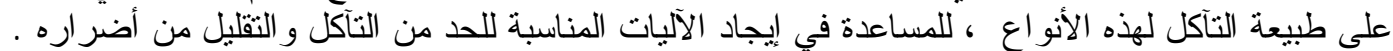

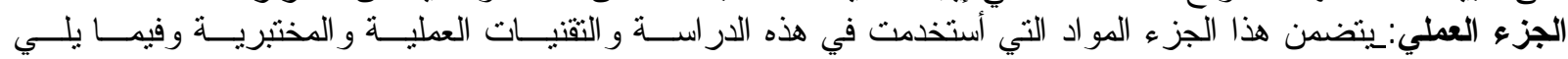

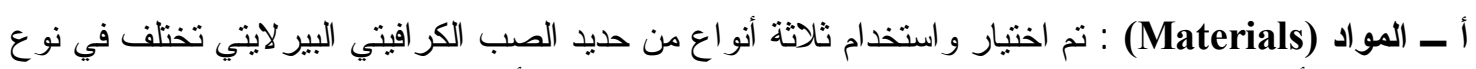

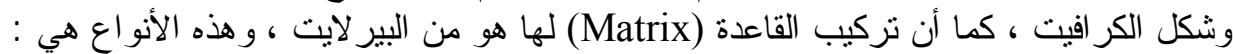

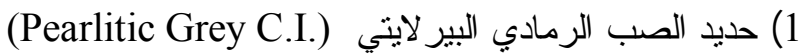

2) حديد الصب الكروي البير لايتي (Pearlitic Spheroidal C.I.) حلايدي الزبي (Pearlitic)

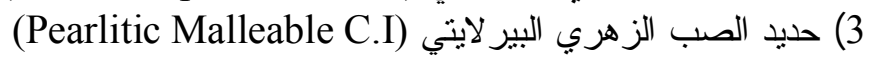
و الجدول (1) يوضح الثركيب الكيمائي (Chemical Composition) للأنو اع المستخدمة في هذا البحث .

$$
\text { الجدول (1) : يتضمن تحليل التركيب الكيمائي لأنو اع حديد الصب الكر افيتي البير لايتي }
$$

\begin{tabular}{|c|c|c|c|}
\hline \multirow{2}{*}{$\begin{array}{c}\text { Chemical } \\
\text { Composition } \\
\text { (Weight \%) }\end{array}$} & \multicolumn{3}{|c|}{ Types of cast iron } \\
\hline & $\begin{array}{c}\text { Pearlitic Gray } \\
\text { C.I. }\end{array}$ & $\begin{array}{c}\text { Pearlitic } \\
\text { Spheroidal C.I. }\end{array}$ & $\begin{array}{c}\text { Pearlitic } \\
\text { Malleable } \\
\text { C.I. }\end{array}$ \\
\hline $\mathrm{C}$ & $\sim 4.65$ & 3.61 & 3.72 \\
\hline $\mathrm{Si}$ & 2.35 & 2.63 & 0.68 \\
\hline $\mathrm{Mn}$ & 0.630 & 0.18 & 0.350 \\
\hline $\mathrm{P}$ & 0.0440 & 0.0290 & 0.0940 \\
\hline $\mathrm{S}$ & $\sim 0.248$ & 0.010 & 0.258 \\
\hline $\mathrm{Cr}$ & 0.109 & 0.180 & 0.230 \\
\hline $\mathrm{Ni}$ & 0.0380 & 0.0840 & 0.0350 \\
\hline Mo & 0.00460 & 0.0301 & 0.0042 \\
\hline $\mathrm{Al}$ & 0.00530 & 0.0110 & 0.0084 \\
\hline $\mathrm{Cu}$ & 0.0920 & 0.173 & 0.0740 \\
\hline $\mathrm{Fe}$ & Balance & Balance & Balance \\
\hline
\end{tabular}

\section{ب - التقتيات العملية (Experimental Techniques) :}

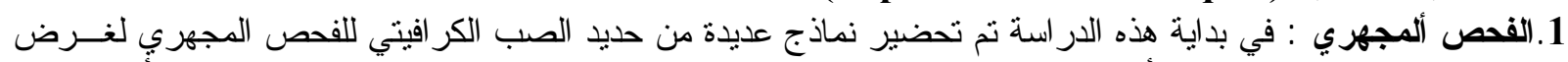

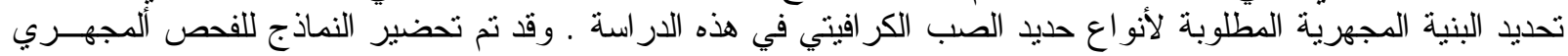

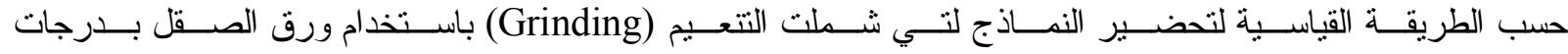

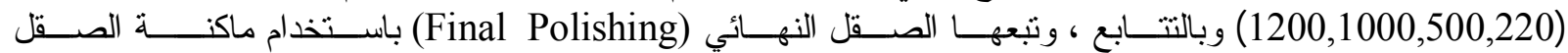

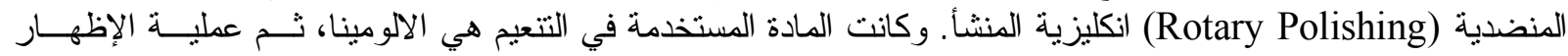

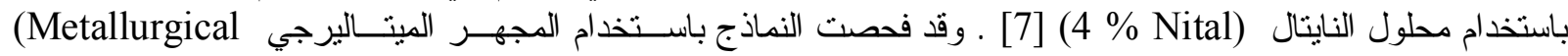

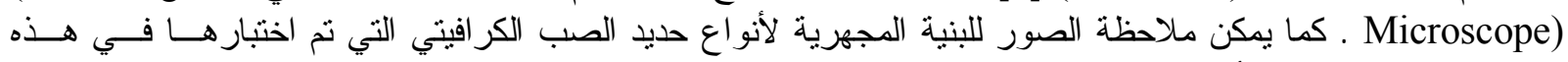

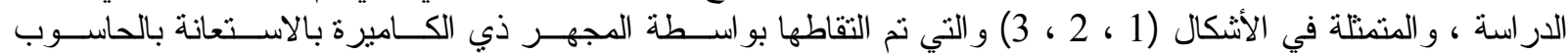



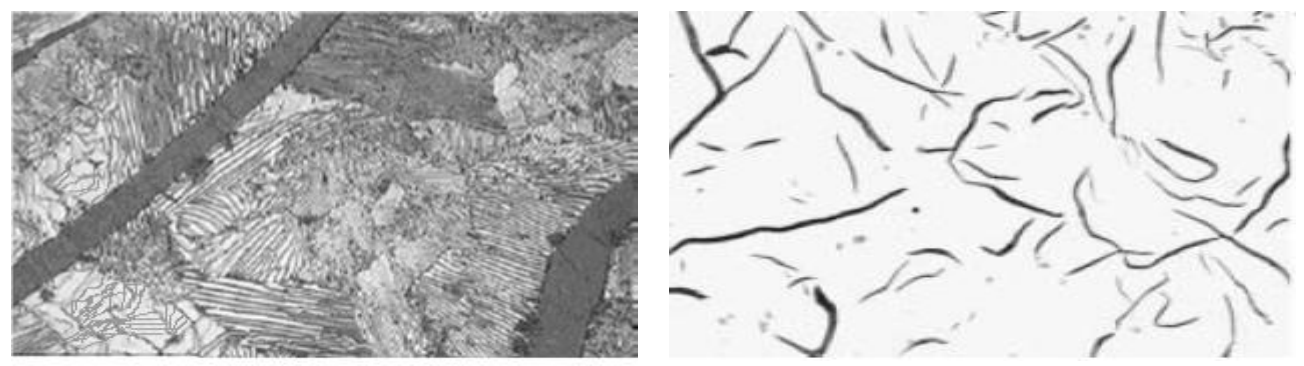

X500 (Etched) X100 (Unetched) البنية المجهرية X الثكل (1) :البنية المجهرية لحديد الصب الرمادي البير لايتي

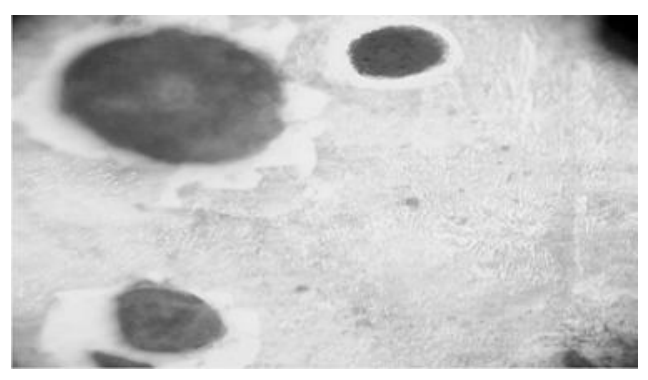

البنية المجهرية (Etched) الثكل (2) :البنية المجهرية لحديد الصب الكروي الكر افيت البير لايتي

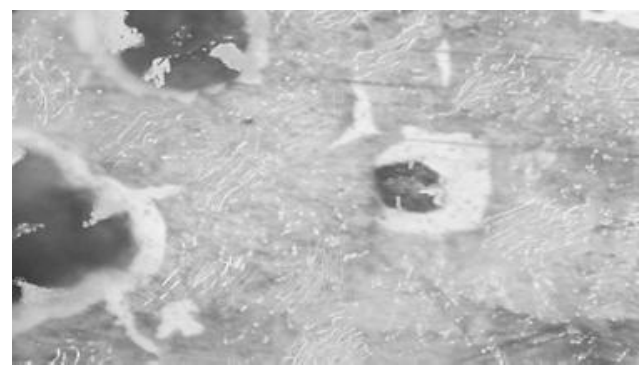

البنية المجهرية X500 (Etched) :البنية المجرية لحديد الصب الزهري الكر افيت البير لايتي
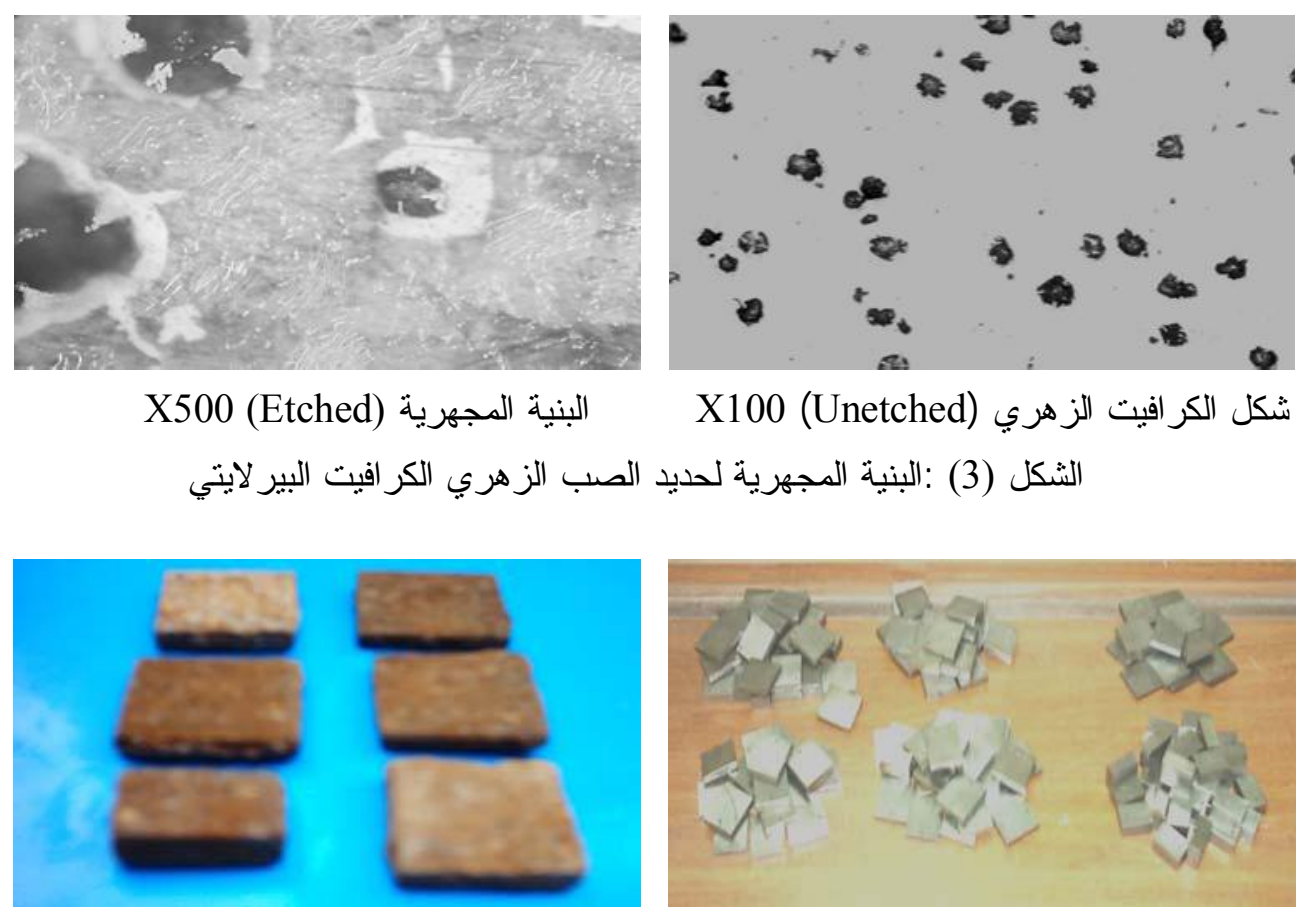

الثكل (5) : عينات من حديد الصب الكر افيتي المتآكلة في التربة الرملية المنب
الثكل (3) : النز

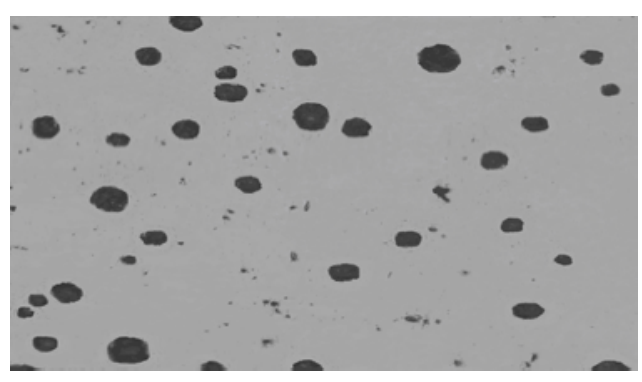

X100 (Unetched) شكل الكر افيت الكروي

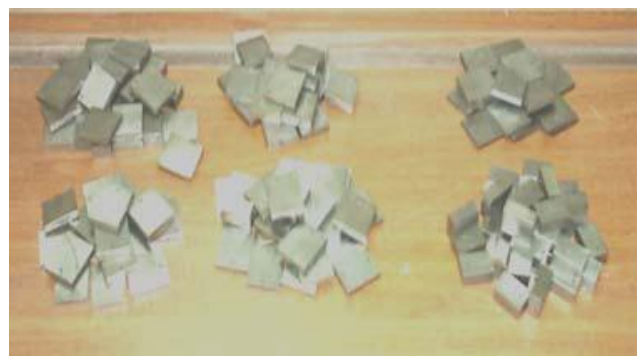

الثنكل (4) : عينات من حديد الصب الكر افيتي قبل إجر اء الاختبار 


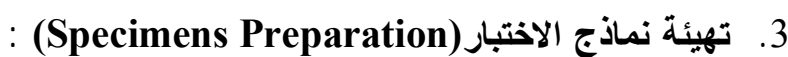

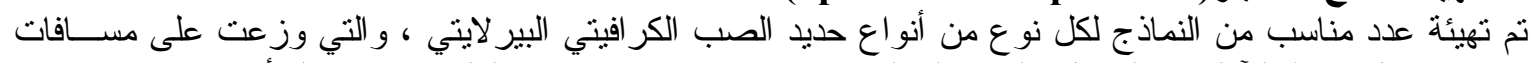

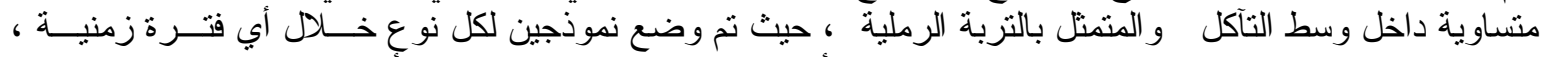

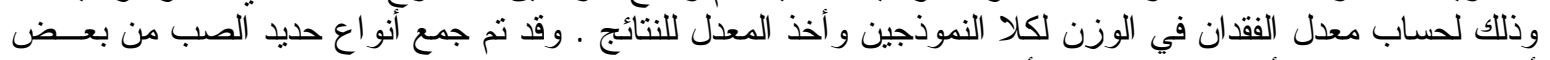

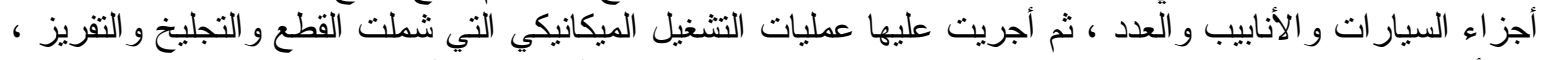

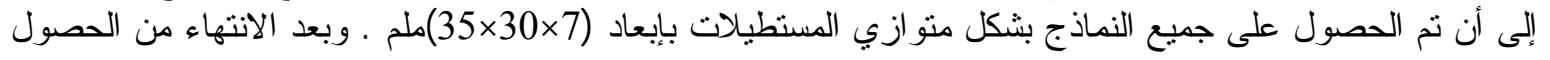

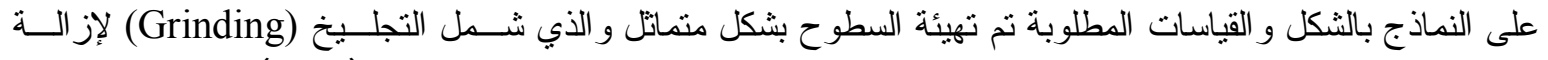

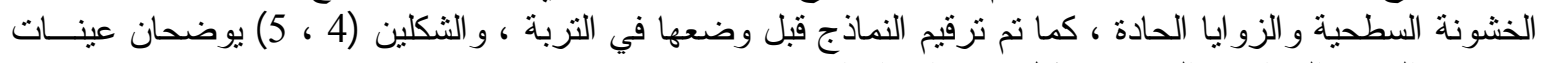

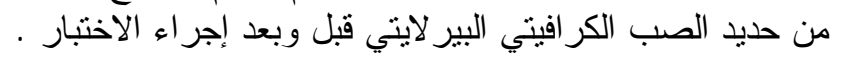

\section{الطريقة المستخدمة لإيجاد معدلات التآكل

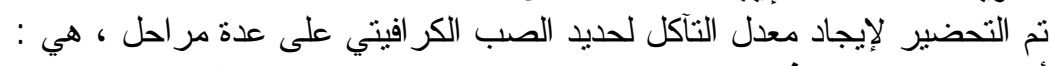

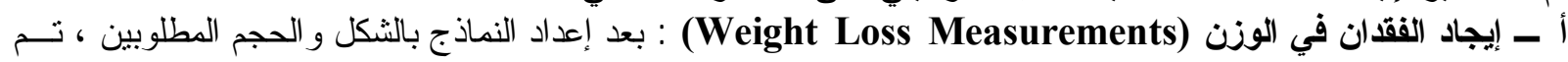

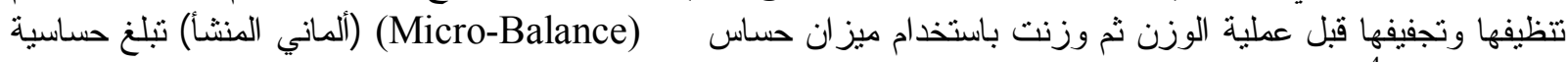

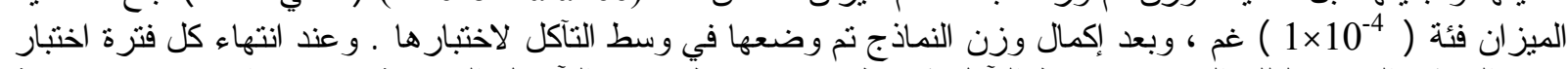

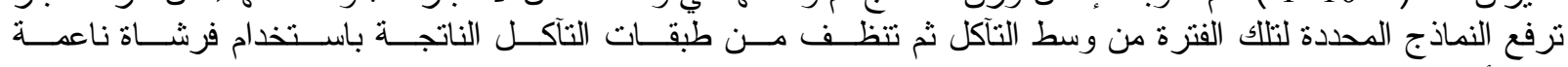

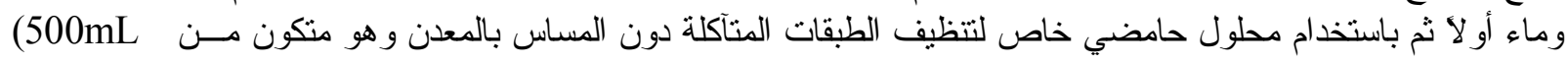
Hcl+3.5g Hexamethylenetetramine +Water to make 1000mL)

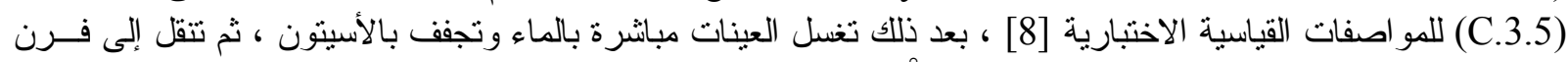

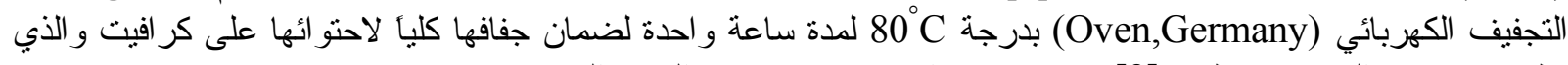

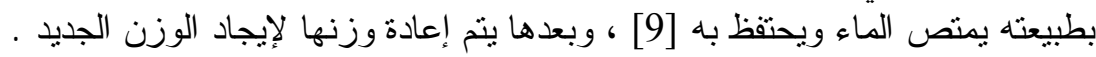

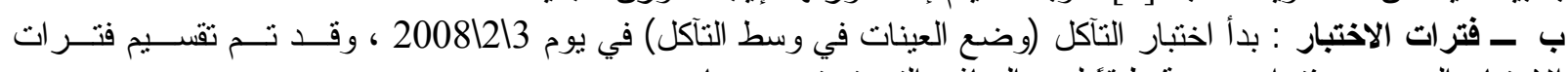

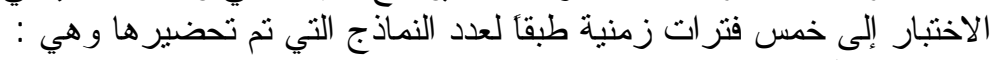

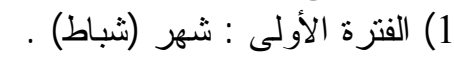

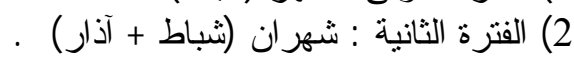

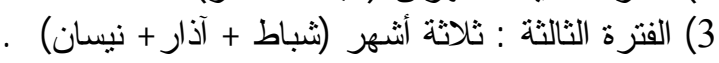
4) الفترة الر ابعة : أربعة أثنهر (شباط (

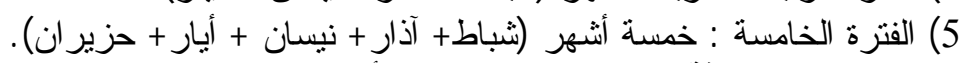

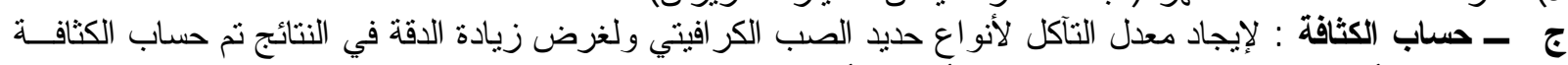

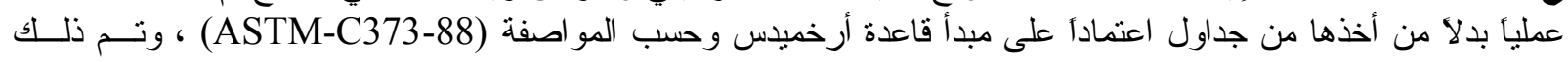

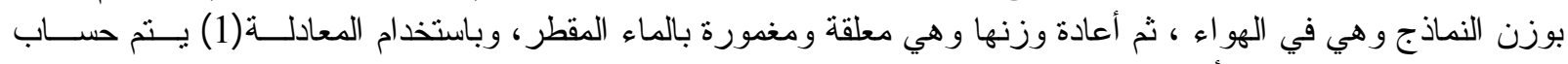

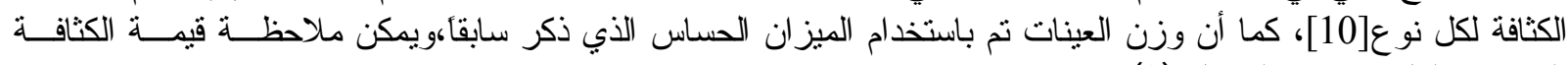

$$
\mathrm{D}=\left[\mathrm{M}_{\mathrm{d}} / \mathrm{M}_{\mathrm{w}}\right] \mathrm{D}_{\mathrm{w}}
$$

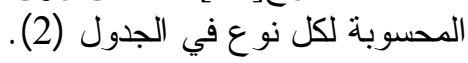

( g/cm³ (الكثافة المحسوبة (المطلوبة) : D

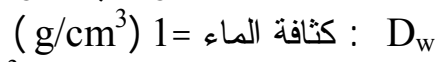
( $\mathrm{cm}^{3}$ (وزن النموذج في الماء (g) وزن النموذج في الهوذج في : وزاء : M 
الجدول (2) : قيم الكثافة المحسوبة لأنو اع حديد الصب الكر افيتي

\begin{tabular}{|l|c|}
\hline \multicolumn{1}{|c|}{ Types of cast iron } & Density $\left(\mathrm{g} / \mathrm{cm}^{3}\right)$ \\
\hline Pearlitic Gray C.I. & 7.1133 \\
\hline Pearlitic Spheroidal C.I. & 7.1556 \\
\hline Pearlitic Malleable C.I. & 7.1266 \\
\hline
\end{tabular}

لد ــ إيجاد معدل التآكل (Corrosion Rate) : تم حساب معدل التآكل لحيد الصب الكر افيتي حسب المو اصفة القياسـية

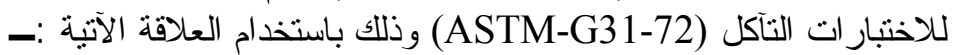

\section{$\left[\frac{534 W}{D A T}\right]=$ Corrosion rate ( mpy)}

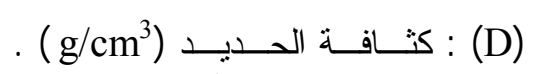

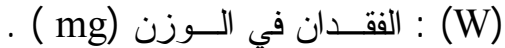

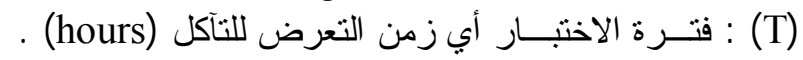

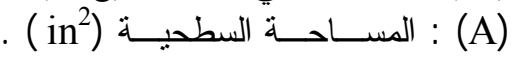
. inch 0.001 تعاد : (mils)

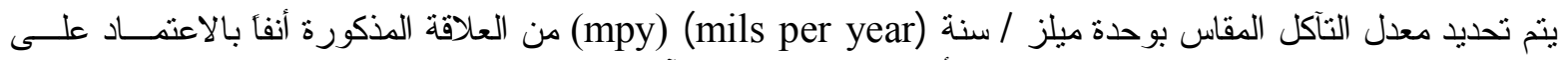

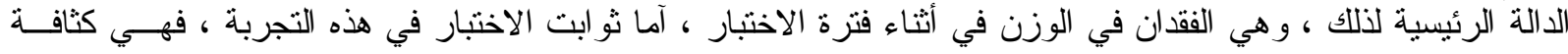

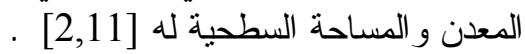

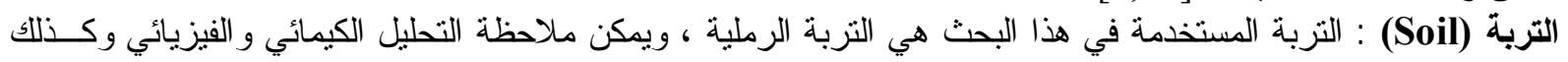

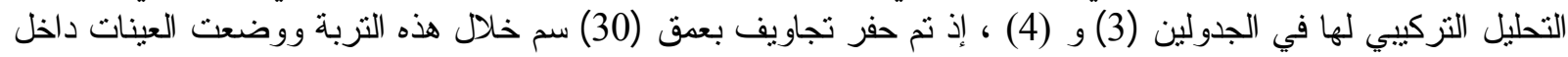

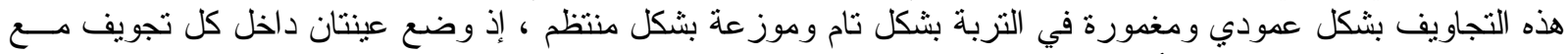

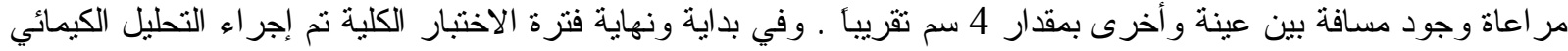

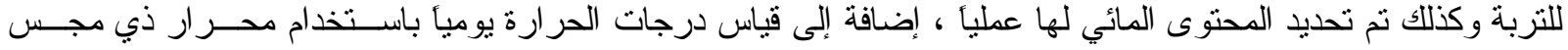

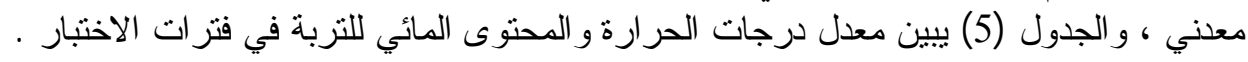

الجدول (3) :التحليل الكيمائي و الفيزيائي للتربة المستخدمة كوسط للتآكل

\begin{tabular}{|l|c|c|}
\hline Soil Quality Parameters & As Prepared & After 5 Months \\
\hline Ec $(\mu \mathrm{s} / \mathrm{cm})$ & 200 & 98 \\
\hline $\mathrm{PH}$ & 8.44 & 8.72 \\
\hline Total Hardness mg/L & 800 & 815 \\
\hline Calcium Hardness mg/L & 720 & 740 \\
\hline Calcium $\left(\mathrm{Ca}^{+2}\right) \mathrm{mg} / \mathrm{L}$ & 288.6 & 242 \\
\hline Magnesium $\left(\mathrm{Mg}^{+2}\right) \mathrm{mg} / \mathrm{L}$ & 17.92 & 16.8 \\
\hline Chlorides $\left(\mathrm{Cl}^{-}\right) \mathrm{mg} / \mathrm{L}$ & 43.98 & 25.7 \\
\hline Sulphates $\left(\mathrm{SO}^{-2}\right)$ & 215.22 & 432.6 \\
\hline $\mathrm{HCO}_{3}{ }^{-}$ & 205.3 & 211 \\
\hline Resistivity $(\Omega . c m)$ & 5000 & 10204 \\
\hline
\end{tabular}


الجدول (4) :تحليل مكونات التربة المستخدمة كوسط للتآكل

\begin{tabular}{|c|c|c|c|}
\hline \multirow{2}{*}{ Contents analysis } & \% Sand & \% Silt & \% Clay \\
\cline { 2 - 4 } & 85 & 10 & 5 \\
\hline
\end{tabular}

الجدول (5) : نسبة المحتوى المائي ودرجة الحرارة

\begin{tabular}{|c|c|c|c|c|c|}
\hline حزير ان & أيار & نيسان & آذار & شباط & زمن التعرض (month) \\
\hline 12 & 17 & 22 & 27 & 28 & المحتوى المائي (\%) \\
\hline 32 & 27 & 24 & 19 & 13 & معدل درجة الحر ارة (م) \\
\hline
\end{tabular}

النتائج و المناقشة التشة أ ـ شكل ونوع الكر افيت وتأثيره على التآكل في حديد الصب الكر افيتي فئي

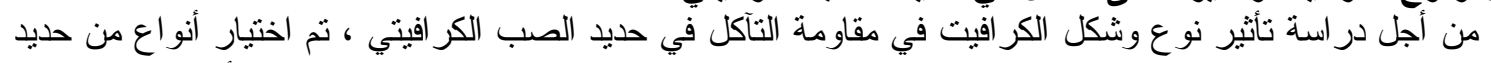

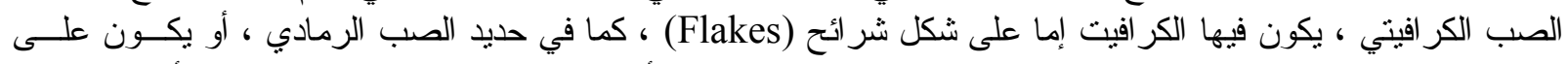

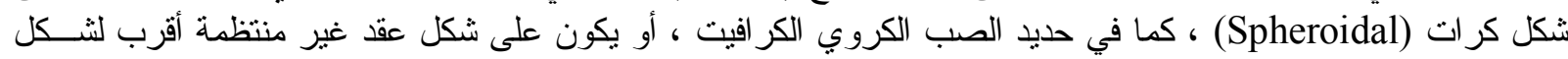

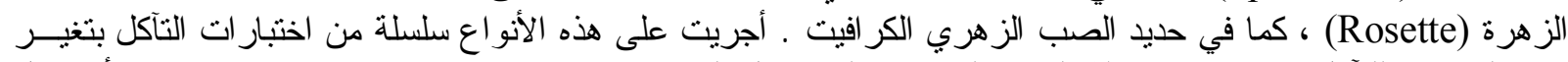

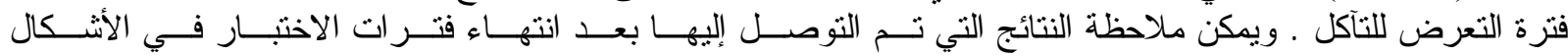
. (9)،(8)،(7)،(6)

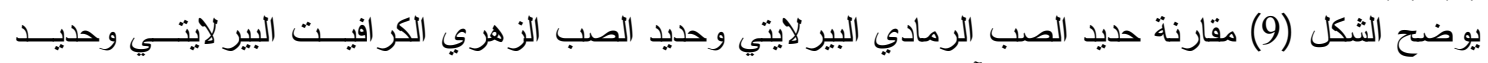

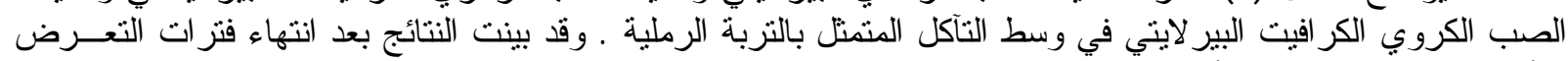

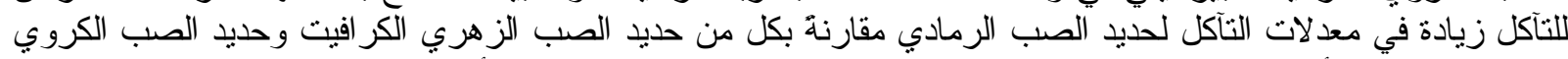

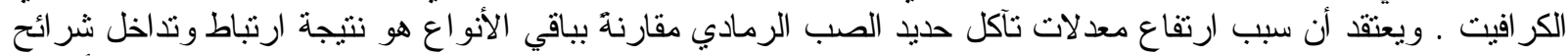

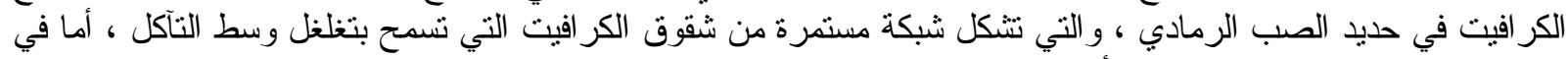

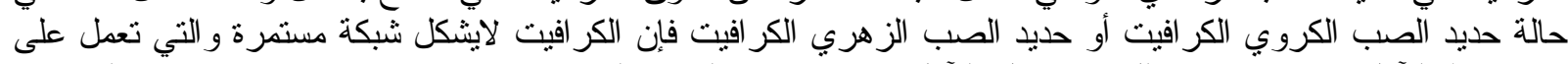

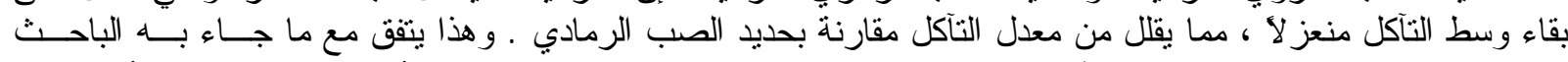

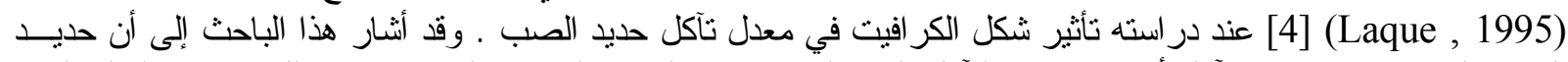

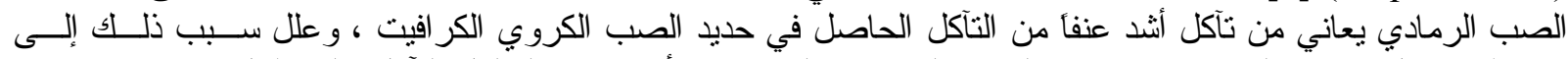

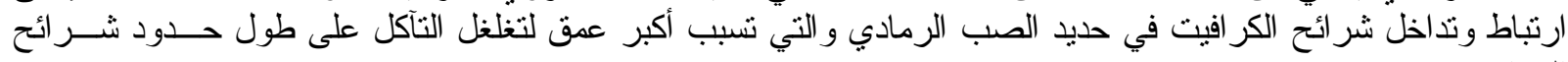

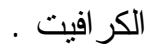

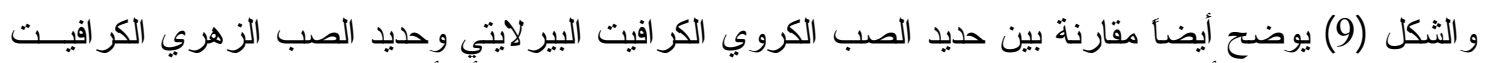

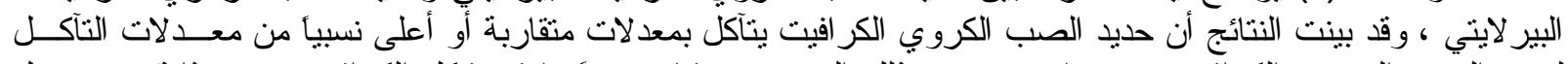

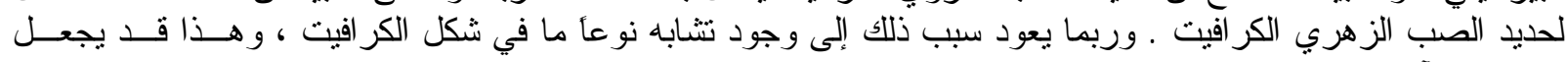

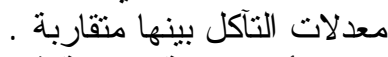

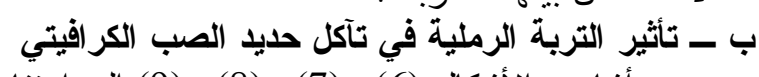

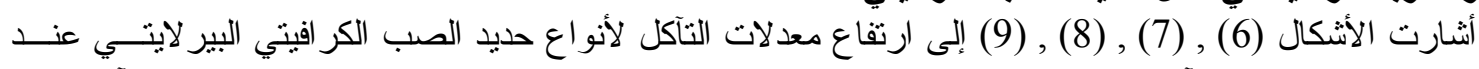

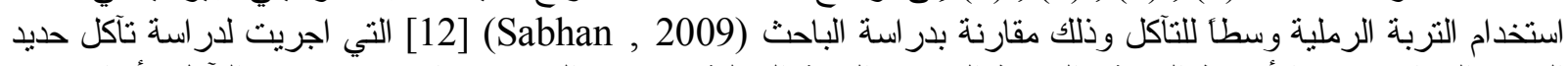

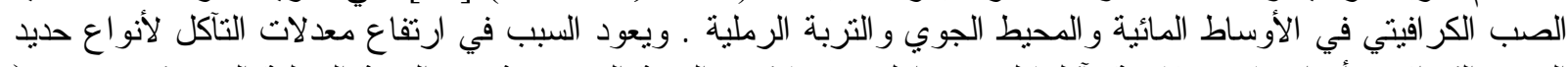

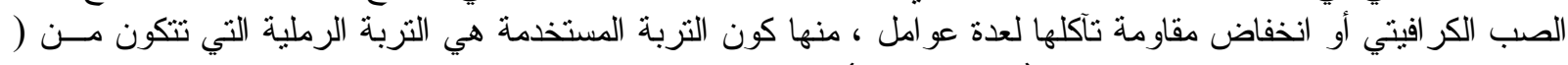

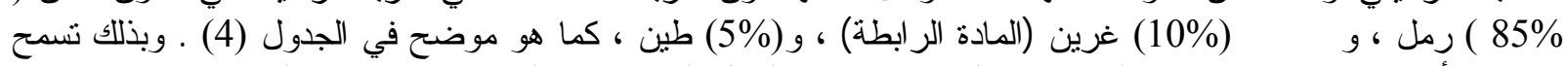

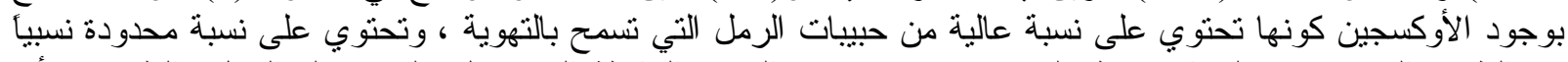

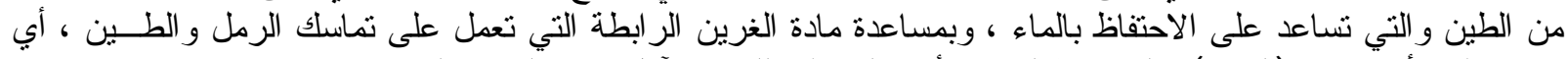

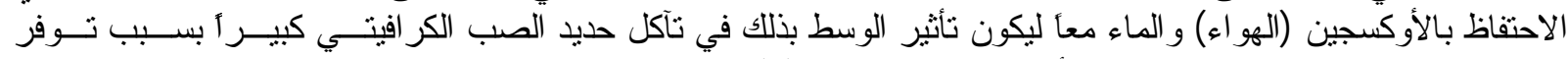

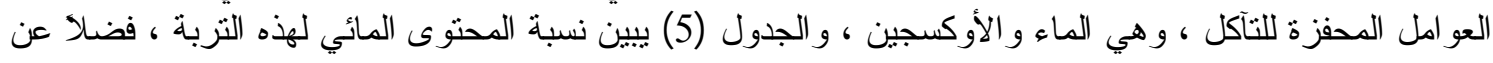




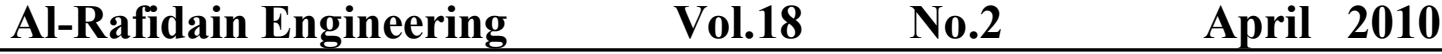

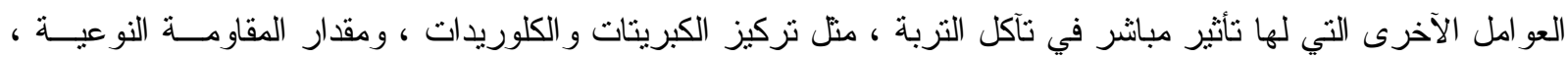

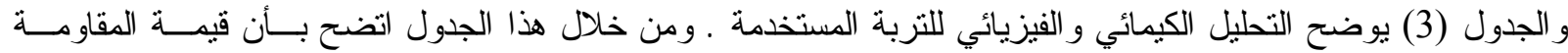

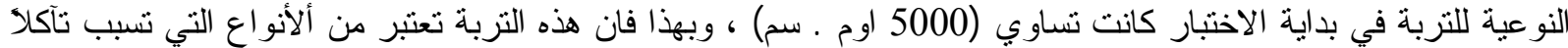

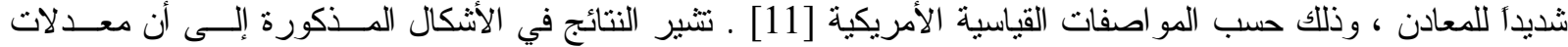

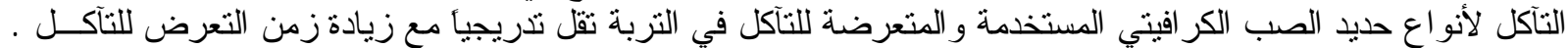

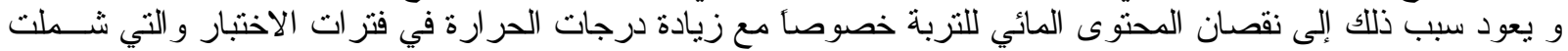

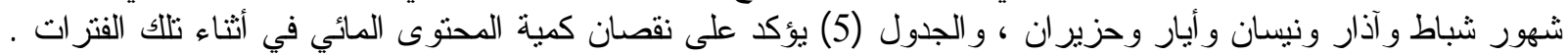

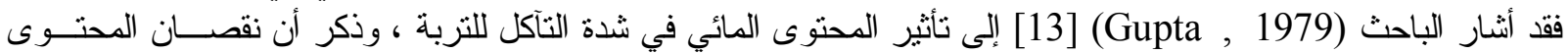

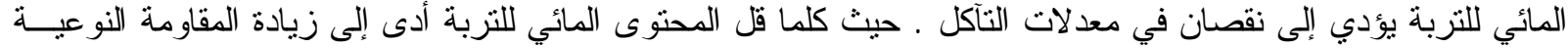

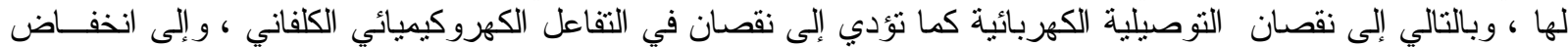

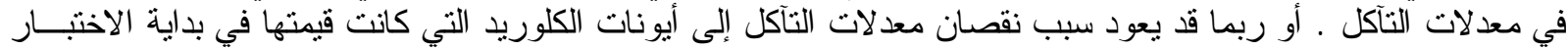

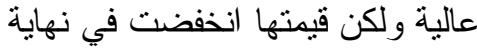

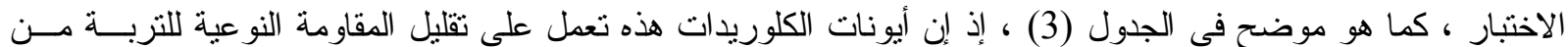

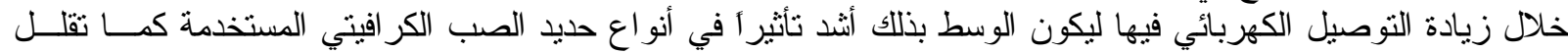

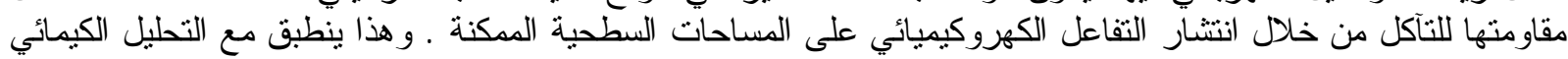

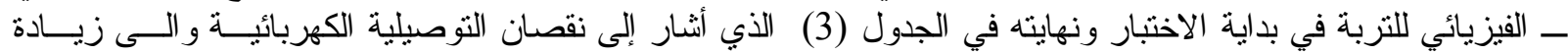
المقاومة النو عية في نهاية الاختبار

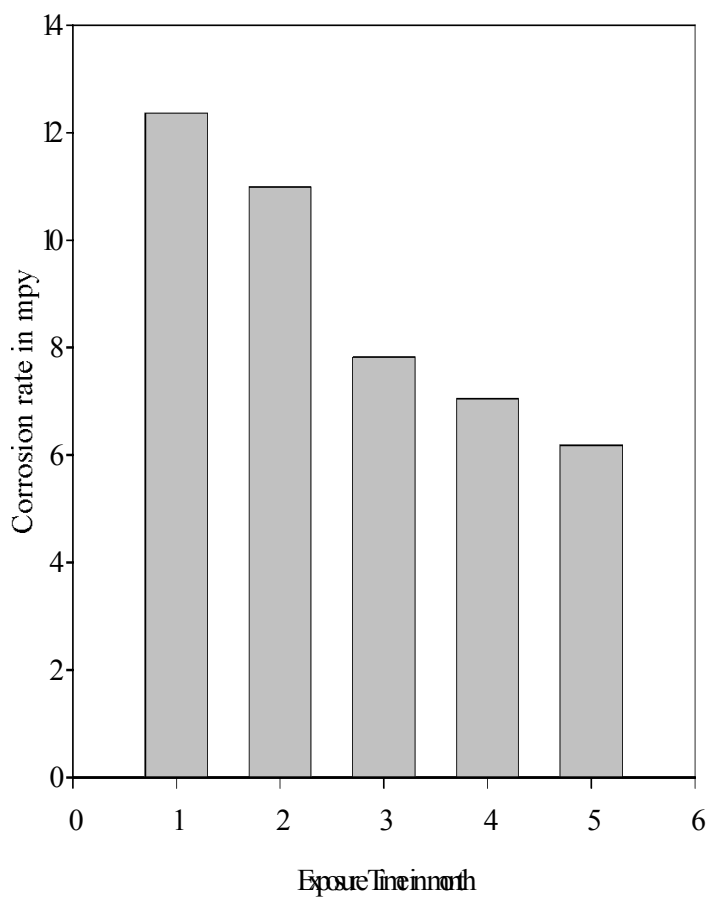

الثكل (6) : معدلات تآكل حديد الصب الرمادي البير لايتي

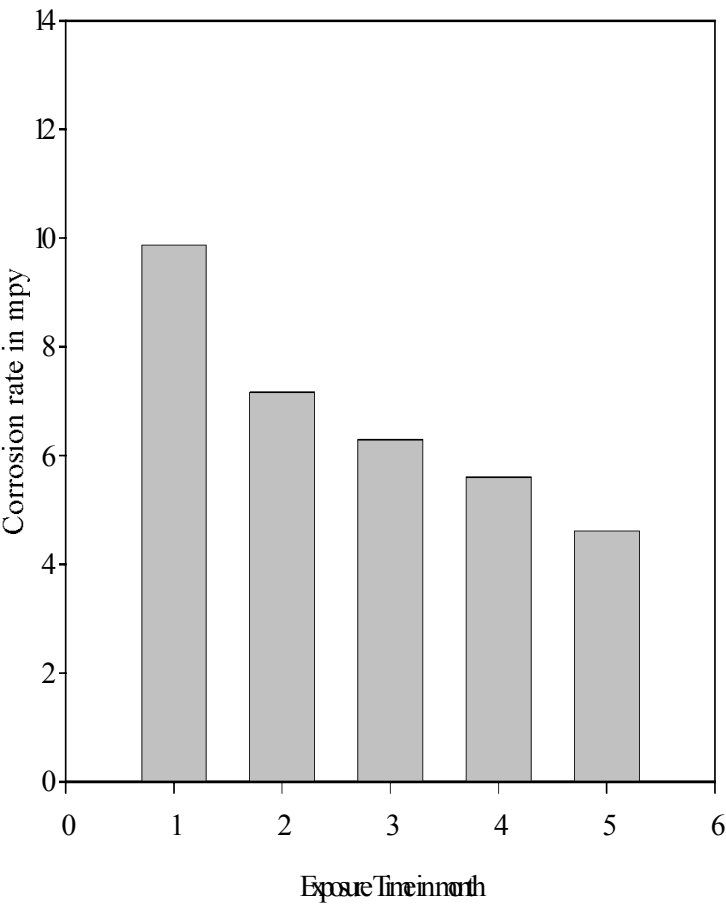

الثكل (7) : معدلات تآكل حديد الصب الكروي الكر افيت البير لايتي الالبير لايتيالبير لايتي 
ابراهيم : دراسة تآكل حديد الصب الكرافيتي البيرلايتي في التربة الرملية

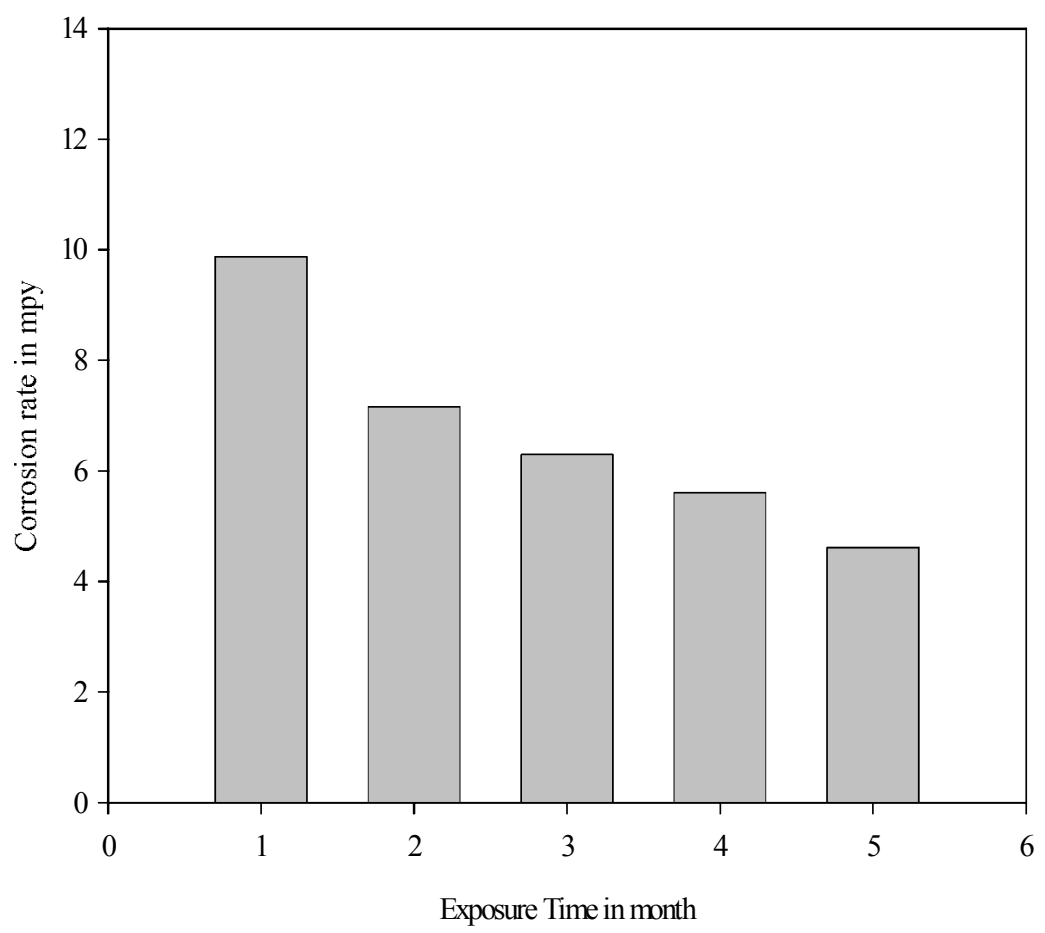

الثكل (8) : معدلات تآكل حديد الصب الزهري الكر افيت البير لايتي

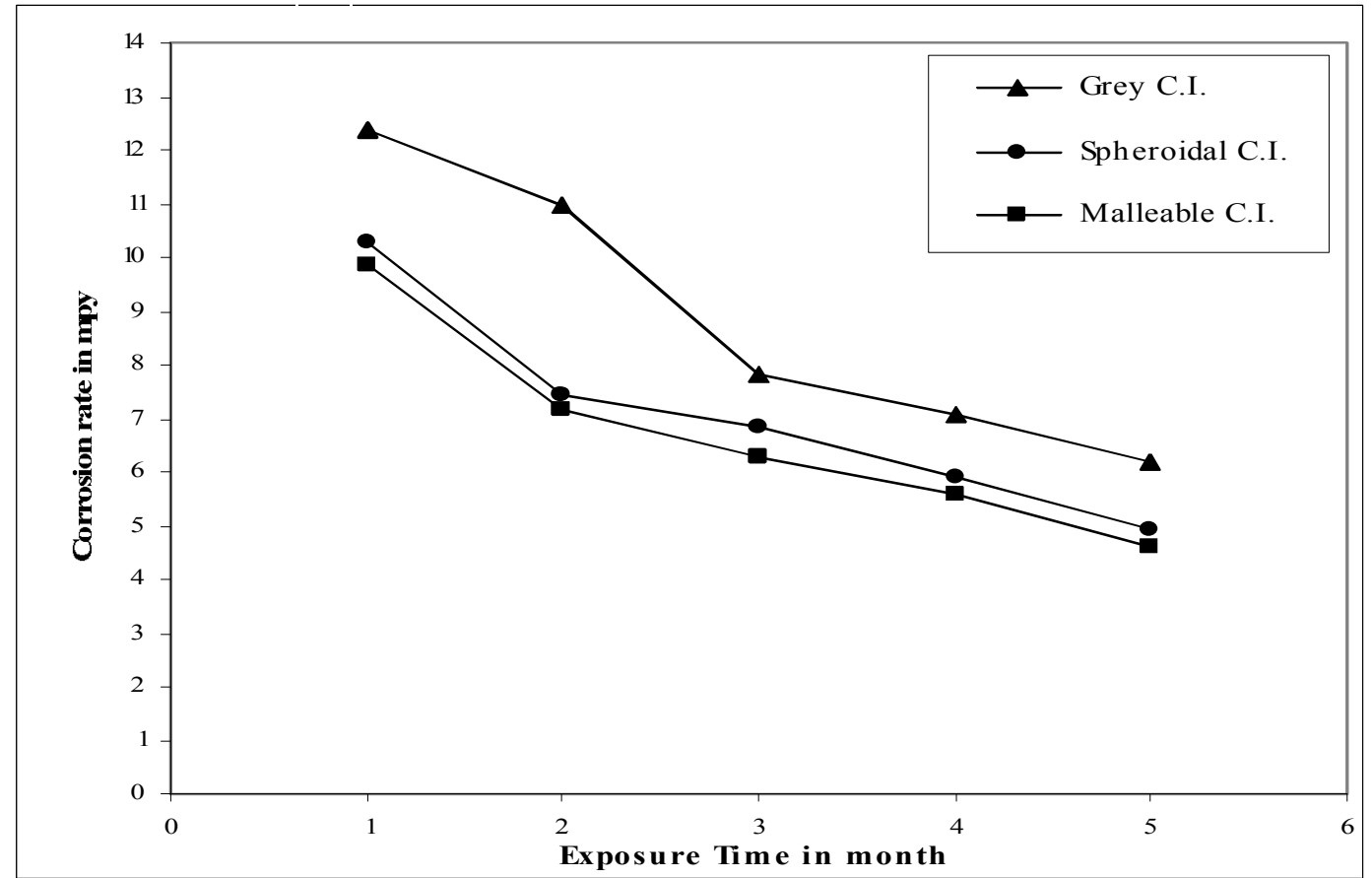

الشكل (9):المقارنة بين معدلات التآكل لأنو اع حديد الصب الكر افيتي البير لايتي في التربة الرملية 


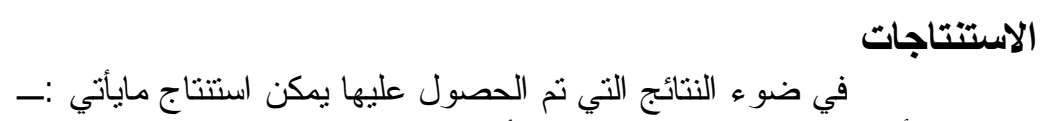

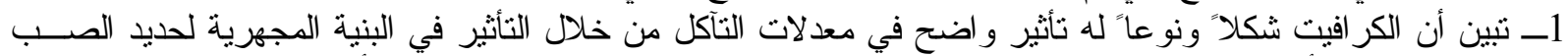

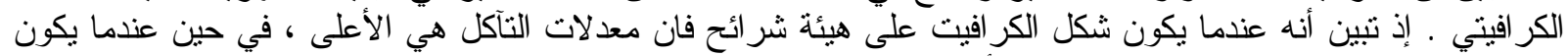

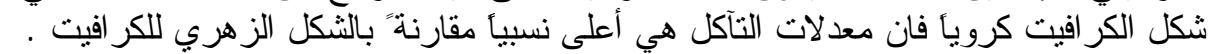

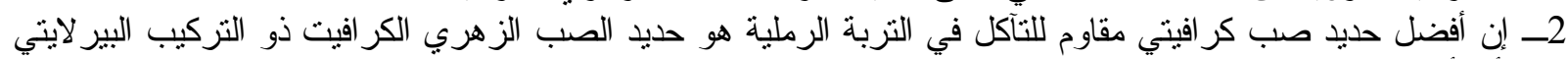

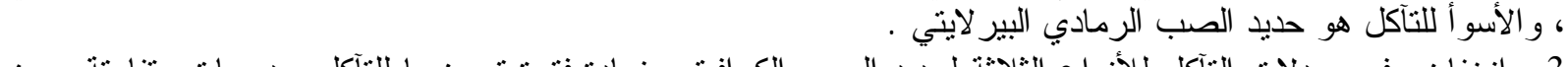

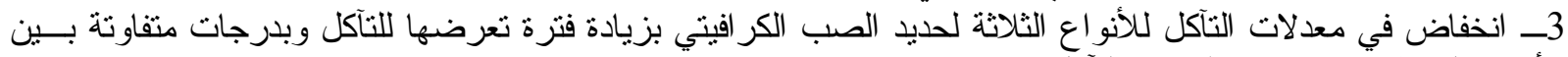

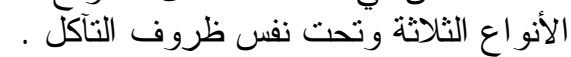

[1] R.Winston R., " Uhlig Corrosion Handbook " , John Wiley \& Sons , Inc, $2^{\text {nd }}$ Edition, New York , (2000), PP. 3-343.

[2] Fontana M.G. , " Corrosion Engineering " , Mc Graw -Hill , $3^{\text {rd }}$ Edition , New York , (1986), PP. 4-173.

[3] Fuller A. G. , "Corrosion Resistance of Ductile Iron Pipe " , BCIRA Report 1442, USA, (1981),PP.2 .

[4] Laque F. , " The Corrosion Resistance of Ductile Iron ", NACE International , November , (1995), PP.1 .

[5] Owate I. \& Arwiri G. , " Impact Of Environmental Conditions on Sub - Surface Storage Tanks (part I) ", Applied Sciences \& Environmental Management, Vol. 6 , NO. 2 , (2002), PP. 79-83 .

[6] Ceki H. " Physiochemical Characteristics of Controlled Low Strength Materials Influencing The Electrochemical Performance and Service Life of Metallic Materials " , Ph. D. , Thesis , University of Texas A \& M, Civil Engineering, (2005), PP. 84 .

[7] Janina M.R. , " Effect of Specimen Preparation on Evaluation of Cast Iron Microstructures " , Materials Characterization, Vol. 54 , (2005), PP. 287-304 .

[8] ASTM Designation : $\mathrm{G}_{1}-90$, " Standard Practice for Preparing, Cleaning, and Evaluating corrosion Test Specimens ", 100 Bar Harbor Drive, West Conshohocken, PA 19428-2959, United States , (1999), PP.5 .

[9] Cao X. \& Xu C. C. , " Effect of Chloride on The Atmospheric Corrosion of Simulated Artifact Iron in $\mathrm{NO}_{3}-$ Bearing Pollutant Environment ", ACTA METALLURGICA SINICA ENGLISH LETTERS , Vol. 19 , NO. 1 ,(2006), PP. 34-42 .

[10] Ted A. , " Fabrication and Damping Behavior of Particulate $\mathrm{BaTio}_{3}$ Ceramic Reinforced Copper Matrix Composites " , M.Sc. ,Thesis ,University of Virginia Polytechnic Institute and State , USA , (2004) , PP. 45-46.

[11] Lawrence J. Korb," ASM Handbook " , Rockwell International and David L Olson , Colorado School of Mines, $9^{\text {th }}$ Edition, USA, (1992), PP. $112-336$.

[12] Sabhan H. A. ," Corrosion Behaviour Investigation of Graphite Containing Cast Irons as Composite-Material Using Local Selected Corrosion Environments " , M.Sc. ,Thesis ,University of Mosul , (2009), PP.72

[13] Gupta S.K. , "The Critical Soil Moisture Content in The Underground Corrosion of Mild Steel ", Corrosion Science, , Vol. 19, (1979) , PP. 171-178.

$$
\text { تم اجراء البحث في كلية الهنلسة - جامعة الموصل }
$$

